\title{
Performance Evaluation Of Parallel TRANSMISSION VISIBLE LIGHT COMMUNICATION SYSTEMS USING COLOUR SHIFT KEYING
}

\author{
Tadahiro Wada $^{1}$, Haruki Furukawa ${ }^{1}$ and Kaiji Mukumoto ${ }^{2}$ \\ ${ }^{1}$ Faculty of Engineering, Shizuoka University, Hamamatsu, Japan \\ ${ }^{2}$ Division of Technical Service, Shizuoka University, Hamamatsu, Japan
}

\begin{abstract}
In this paper, we deal with parallel-transmission visible light communication (PT-VLC) systems using Colour-Shift Keying (CSK). We construct a prototype of PT-VLC systems utilizing a Liquid Crystal Display $(L C D)$ as a transmitter and an Image Sensor (IS) as a receiver. We also propose a new colour estimation algorithm for CSK and integrate the algorithm into the prototype. We show effectiveness of the system by evaluating Bit Error Rate (BER) performance, Frame Error Rate (FER) performance, and throughput performance. We also adopt a Turbo code for error correction and show its effectiveness in the proposed VLC system.
\end{abstract}

\section{KEYWORDS}

Visible light communication, Colour shift keying, Bit error rate performance, Turbo code

\section{INTRODUCTION}

Visible Light Communication (VLC) is a communication scheme using visible light as a carrier [1]-[14]. VLC has many advantages compared with wireless radio communications :VLC has little influence on human bodies and precision equipment; it can be used in places where wireless communication by radio waves is prohibited. Furthermore, by using VLC, we can keep away from the Radio law for wireless radio communications.

Most of past studies of VLC adopt light emitting diodes (LEDs) as a light transmission device and photo diodes as a receiving device. VLC systems using LEDs and photo diodes have high capabilities of achieving high-data-rate transmission such as several hundred Mbps.

In recent years, we have been witnessing a significant increase of Liquid Crystal Displays (LCDs) because of rapid spreading of personal computers, TVs and smart phones. For this reason, transmitters using LCDs have received much attention as alternative VLC systems. One benefit of the LCDs is easy brightness control of three colours. We can expect the LCDs to have high flexibility for displaying images/movies including data information.

For the VLC systems using LCDs, image sensors (ISs) are usually used for light receiving equipment [5]-[9]. ISs are also popular because they are equipped with digital cameras and smart phones. One advantage of ISs compared with PDs is that ISs have a wide angle of view and do not require strict position settings. And other advantage is robustness to optical disturbance. Although PDs are seriously affected by optical disturbance, ISs can reduce the influence because of their wider angle of view. 
For utilizing ISs for VLC systems, we have to take their low flame rate into account. The flame rate of popular ISs is 30-60 fps and the low flame rate completely limits the transmission rate of VLC systems. One solution is to adopt Parallel Transmission (PT) by which large numbers of information light sources are transmitted. Since the ISs have high directionality and resolution, spatial division can be achieved for the detection of parallel signals $[8,9]$.

Colour Shift Keying (CSK) is an alternative technique to increase a transmission rate. Using simple ON-OFF keying, only one bit can be transmitted by one light source. However, when we can use three primary colours, red, green, and blue, one light source can transmit three-bit information. VLC systems using LCDs are suited for applying CSK because light strength of three primary colours of LCDs is easily controllable.

Previous works that deal with CSK for VLC systems utilize colour LEDs[10]-[13]. For example, Ref. [10] deals with improvement of signal constellation by using finite state machines. In [11], the authors evaluate communication performance by using multi-colour LEDs. Most of previous works about CSK utilize LEDs and there are few works about CSK using LCDs. A famous application system of CSK using LCDs is explained in [14]. The system does not adopt parallel transmission and it archives very low transmission rate. Therefore, we need more efforts to study about LCDs based VLC systems using CSK.

In this paper, we construct a prototype of PT-VLC systems which utilizes an LCD as a transmitter and an IS as a receiver. We also propose a new colour estimation algorithm for CSK and integrate the algorithm into the prototype. We show the effectiveness of the system by evaluating Bit Error Rate (BER) performance, Frame Error Rate (FER) performance and throughput performance. We also adopt a Turbo code for error correction[15]. We show effectiveness of the Turbo code in the proposed VLC system.

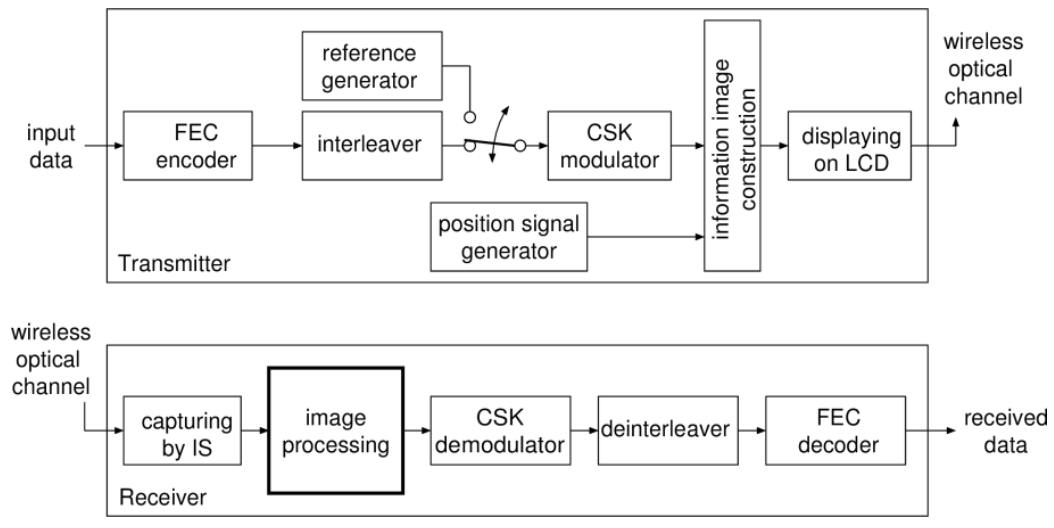

Figure 1: Block diagram of the prototype of PT-VLC systems

\section{SYSTEM CONSTRUCTION}

This section describes a transmitter and a receiver of the prototype of PT-VLC systems using CSK. Figure 1 shows the block diagram of the prototype

The system transmits digital information by displaying colours on an LCD. Since information is continuously transmitted by changing colours on successive frames of the LCD, we can see information signal on the LCD as a moving image.

The receiver uses an IS for capturing a moving image and detects the information by using the proposed colour estimation algorithm. We need to set the frame rate of the IS as the same as that of the LCD. 


\subsection{Behaviour Of The Transmitter}

\subsubsection{USED COLOURS AND IMAGE CONSTRUCTION}

At first, data is fed to an FEC encoder followed by an inter leaver. And then, the interleaved data is fed to a CSK modulator and an image signal constructor. An example of a constructed image is shown in Figure 2. The image is then displayed on an LCD. The LCD utilized by this system is shown in Fig. 3(a). The specification of the LCD is described in Table I.

Each pixel on the LCD has three primary colours, red, green, and blue. Three variables, $r, g$ and $b$, denote light intensities of the LCD and have a range from 0 to 255 (corresponding to 8 bits). By mixing these primary colours, we can display various colours. In this study, to simplify evaluation of effect of CSK, we assume that the light intensity of each colour at the LCD is only 0 or 255. Therefore, for data transmission, the LCD emits 8 colours. The set of the colours, $C$, is expressed as,

$$
\text { C=\{Black,Blue,Magenta,Red,Yellow, Green, Cyan, White }\} .
$$

Their light intensities, $(r, g, b)$, can be expressed as, $(0,0,0),(0,0,255),(255,0,255),(255,0,0)$, $(255,255,0),(0,255,0),(0,255,255)$, and $(255,255,255)$, respectively.

As shown in Figure2, in order to achieve parallel transmission, the images consist of $M \times N$ boxes and each box consists of some liquid crystal pixels. Here, we introduce two variables, $m$ and $n$, which indicate the coordinate of each box. The range of $m$ and $n$ is $0 \leq m<M$ and $0 \leq n<N$, respectively. For example, the box at the 3 rd column and at the 0 th row is represented asBOX $\mathrm{X}_{(0,3)}$.

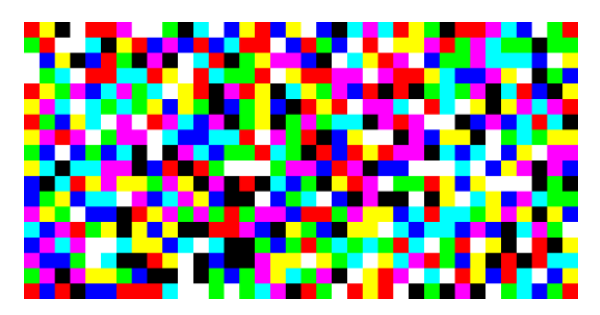

Figure 2: An Example of a constructed image

\subsubsection{POSITION SIGNAL AND COLOUR REFERENCE FRAME GENERATION}

By the receiver, an information area of captured images should be detected. In this system, an Msequence is employed for position detection of the information area[8]. The M-sequence can achieve reliable position detection because it has excellent auto-correlation characteristic. The signals for the position detection are located at four corners, i.e., $\operatorname{BOX}_{(0,0)}, \operatorname{BOX}_{(0, N-1)}$, $\operatorname{BOX}_{(M-1,0)}$, and $\operatorname{BOX}_{(M-1, N-1)}$.

The role of the reference generator in the transmitter is to make a colour reference frame used for colour estimation at the receiver. The colour reference frame is periodically inserted into data frames. We explain how to use the colour reference in the next section in detail.

\subsection{BEHAVIOUR OF THE RECEIVER}

In the prototype, a USB camera (as an IS) shown in Figure3 is used for capturing a displayed image. The specification of the camera is shown in Table 2. 
The International Journal of Multimedia \& Its Applications (IJMA) Vol.9, No.2, April 2017

The captured image is then fed to a personal computer for image processing. The role of the image processing block is to estimate tranmitted colours and we explain behavior of it in the next section.

From estmated colours, received data is obtained by using a CSK demodulator, a de-interleaver, and an FEC decoder.

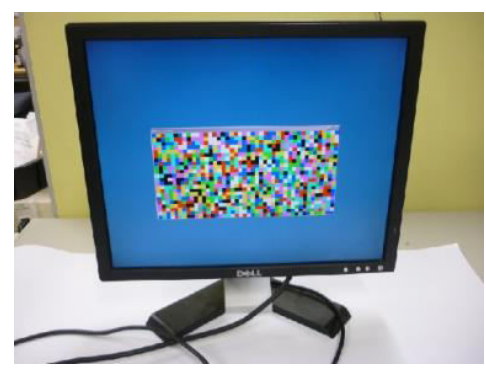

(a) LCD

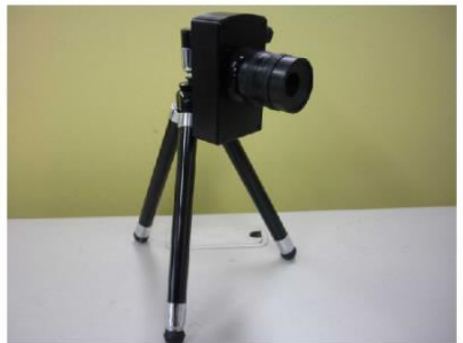

(b) USB camera

Figure 3: Experimental equipment

Table 1: Specifications of the LCD

\begin{tabular}{cc}
\hline \hline Name of product & FTD-G722AS2 \\
\hline Display size & $17[$ inch] \\
\hline Resolution & $1280[$ pixel] $] \times 768[$ pixel] \\
\hline Power consumption & $23[\mathrm{~W}]$ \\
\hline \hline
\end{tabular}

Table 2: Specification of the USB Camera

\begin{tabular}{cc}
\hline \hline Name of product & Lumenera Lu75C \\
\hline Resolution of images & 640 [pixel] $\times 480$ [pixel] \\
\hline Gradation & $8[\mathrm{bit}]$ \\
\hline Maximum frame rate & $60.0[\mathrm{fps}]$ \\
\hline \hline
\end{tabular}

\section{Colour Estimation Algorithm}

One purpose of the paper is to propose a new colour estimation algorithm for PT-VLC systems. Our original brightness estimation algorithm was proposed in [8] for a system utilizing an LED matrix as a transmitter and adopting ON-OFF keying. In this paper, we completely modify the algorithm applicable for colour estimation when an LCD is used as a transmitter. In this section we explain the modified algorithm in detail. Figure 4 shows behavior of image processing block including the colour estimation algorithm.

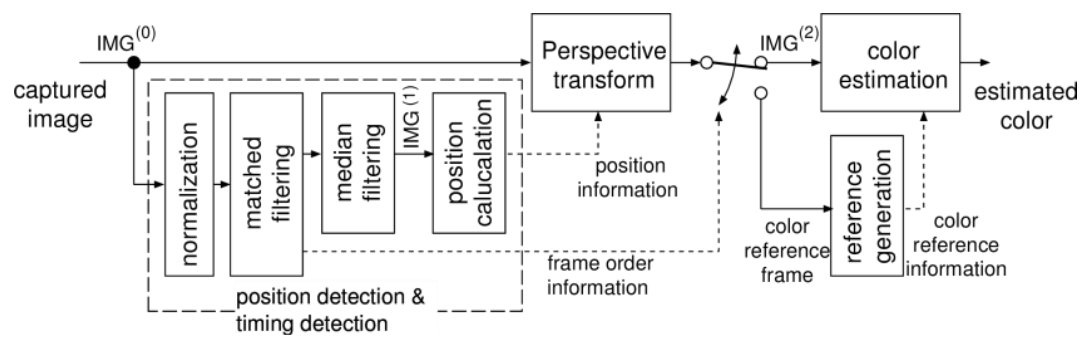

Figure 4: Behavior of the image processing block at the receiver 


\subsection{CAPTURING AN IMAGE BY IS}

The captured image is denoted as $\mathrm{IMG}^{(0)}$. We assume that a captured image has the height $I^{(0)}$ and the width $J^{(0)}$. Figure $5\left(\right.$ a) is an example of $\mathrm{IMG}^{(0)}$, where $I^{(0)}$ is 480 [pixel] and $J^{(0)}$ is 640 [pixel], respectively. In this paper, variables $i$ and $j$ denote the coordinate of pixels of $\mathrm{IMG}^{(\alpha)}$, i.e., the ranges of $i$ and $j$ are $0 \leq i<I^{(\alpha)}$ and $0 \leq j<J^{(\alpha)}$, respectively. Since $\mathrm{IMG}^{(0)}$ is a colour image, each pixel has three colour intensities, i.e., red, green, and blue. These received signal intensities are denoted as $R_{(i, j)}, G_{(i, j)}$, and $B_{(i, j)}$, respectively.

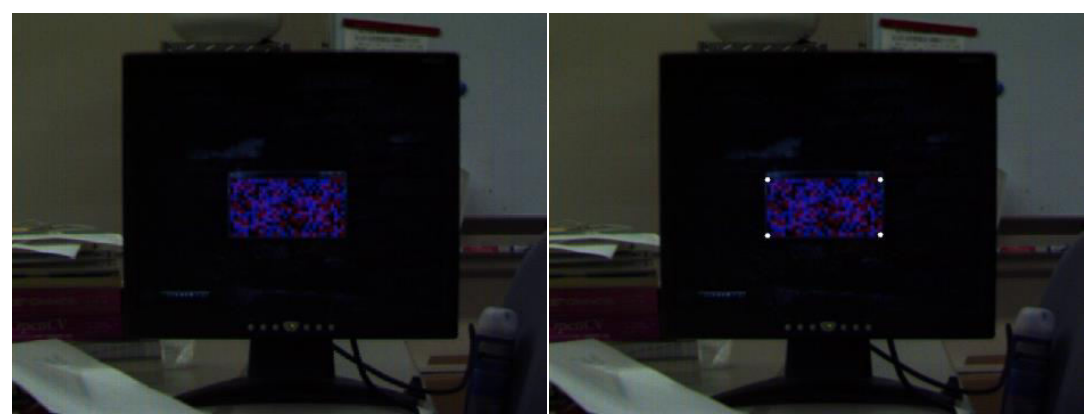

(a) Captured image

(b) Position-detected image

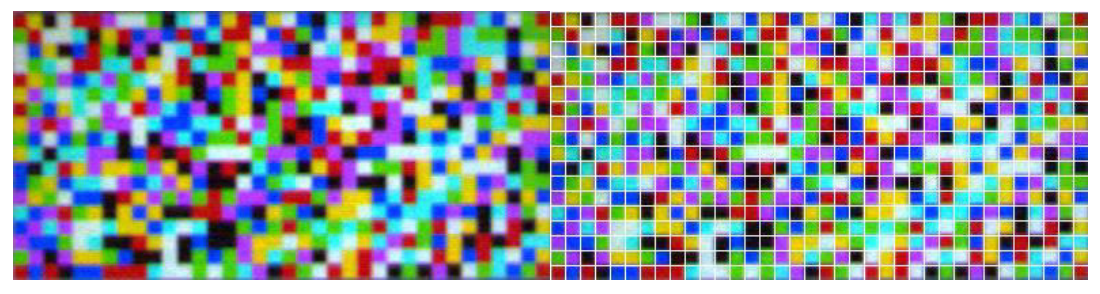

(c) Perspective-transformed image

(d) Partitioned image

Figure 5: Sample images on information processing

\subsection{POSITION DETECTION AND PREAMBLE FRAME DETECTION}

Next, the position of an information area in the captured image should be determined. As mentioned earlier, an M-sequence is used for the detection of the information area. We apply the detection algorithm shown in[8]. In accordance with Ref. [8], a position-detected image is denoted as $\mathrm{IMG}^{(1)}$, which is shown in Fig 5.(b) .

By using the auto-correlation property of the M-sequence, order of displayed frames can be also identified. Therefore, the receiver can detect which frame is a reference frame[8].

\subsection{PeRSPECTIVE TranSFORM}

Position, shape, and size, of an information area in $\mathrm{IMG}^{(0)}$ depend on a relative location between the LCD and the USB camera, that is, the information area is inconstant in every $\mathrm{IMG}^{(0)}$. In order to detect transmitted colours, the information area needs to be transformed to be a fixed-size rectangle. In this system, Perspective Transform is adopted for this transform[8]. The transformed image, $\mathrm{IMG}^{(2)}$, is shown in Fig. 5(c) and its size, $I^{(2)} \times J^{(2)}$, is 640 [pixel] $\times 480$ [pixel] in this study. 


\subsection{Colour Estimation}

At the final step of the image processing block, we estimate transmitted colours of each box. In order to identify all boxes, $\mathrm{IMG}^{(2)}$ is evenly partitioned into $M \times N$ areas as shown in Fig. 5(d). Each partitioned area is named as a "cell" in this paper. The height of a cell, $c_{\mathrm{h}}$, and the width of it, $c_{\mathrm{w}}$, are given by

$$
c_{\mathrm{h}}=\frac{I^{(2)}}{M} \text { and } c_{\mathrm{w}}=\frac{J^{(2)}}{N}
$$

respectively. The cell at the $m^{\text {th }}$ row and the $n^{\text {th }}$ column is denoted as $\operatorname{CELL}_{(m, n)}$. Here, we define signal intensities of cells, $R_{(m, n)}, G_{(m, n)}$, and $B_{(m, n)}$, which are derived by,

$$
\begin{aligned}
R_{(m, n)} & \equiv \frac{1}{c_{\mathrm{h}} c_{\mathrm{w}}} \sum_{i=c_{\mathrm{h}}(m-1)}^{c_{\mathrm{h}} m} \sum_{j=c_{\mathrm{w}}(n-1)}^{c_{\mathrm{w}} n} R_{(i, j)}^{(2)}, \\
G_{(m, n)} & \equiv \frac{1}{c_{\mathrm{h}} c_{\mathrm{w}}} \sum_{i=c_{\mathrm{h}}(m-1)}^{c_{\mathrm{h}} m} \sum_{j=c_{\mathrm{w}}(n-1)}^{c_{\mathrm{w}} n} G_{(i, j)}^{(2)}, \quad \text { and } \\
B_{(m, n)} & \equiv \frac{1}{c_{\mathrm{h}} c_{\mathrm{w}}} \sum_{i=c_{\mathrm{h}}(m-1)}^{c_{\mathrm{h}} m} \sum_{j=c_{\mathrm{w}}(n-1)}^{c_{\mathrm{w}} n} B_{(i, j)}^{(2)},
\end{aligned}
$$

where $R_{(i, j)}^{(2)}, G_{(i, j)}^{(2)}$, and $B_{(i, j)}^{(2)}$, are signal intensities at the coordinate $(i, j)$ of $\mathrm{IMG}^{(2)}$. An example of signal intensities of cells, $\left(R_{(m, n)}, G_{(m, n)}, B_{(m, n)}\right)$, in the RGB colour space is shown in Fig. 6. We found that the intensities of cells having the same colour are concentrated at near eight vertices of corresponding colours. The complexity of caluclation for each cell is approximately $O\left(c_{h} \times c_{w}\right)$.

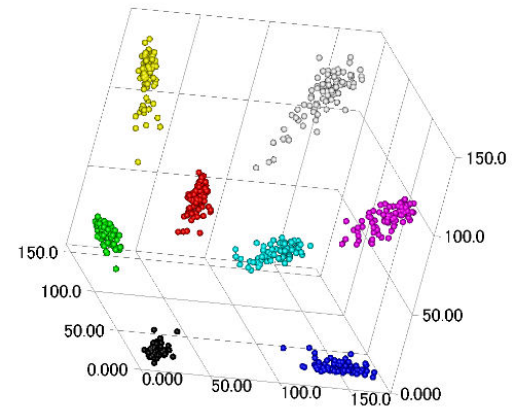

Figure 6: An example of signal intensities for each colour

In order to perform colour estimation of each cell, the Euclid distance $d$ on a colour space is defined as follows,

$$
d_{c(m, n)}=\sqrt{\frac{\left(R_{(m, n)}-R_{r e f}^{c}\right)^{2}}{\left(\sigma_{R, r e f}^{c}\right)^{2}}+\frac{\left(G_{(m, n)}-G_{r e f}^{c}\right)^{2}}{\left(\sigma_{G, r e f}^{c}\right)^{2}}+\frac{\left(B_{(m, n)}-B_{r e f}^{c}\right)^{2}}{\left(\sigma_{B, r e f}^{c}\right)^{2}}},
$$

where $c$ denotes a colour and $c \in C$. Because eight colours are used for the system, eight Euclid distances are calculated for each cell. The receiver selects a colour corresponding to the smallest Euclid distance as the transmitted colour. 


\subsection{Colour References}

$R_{r e f}^{c}, G_{r e f}^{c}$, and $B_{r e f}^{c}$ in Eq. (4) are called as reference intensities for the colourc, where $c \in C$. The reference intensities are initially determined by using a colour reference frame.

We define the set $S_{c}$ as $S_{c}=\left\{(m, n) \mid\right.$ the colour of $\operatorname{CELL}_{(m, n)}$ in a colour reference frame is $\left.c\right\}$. $R_{r e f}^{c}, G_{r e f}^{c}$, and $B_{r e f}^{c}$ can be expressed as,

$$
\begin{aligned}
R_{r e f}^{c} & =\frac{1}{\left|S_{c}\right|} \sum_{(m, n) \in S_{c}} R_{(m, n), r e f}, \\
G_{r e f}^{c} & =\frac{1}{\left|S_{c}\right|} \sum_{(m, n) \in S_{c}} G_{(m, n), r e f}, \text { and } \\
B_{r e f}^{c} & =\frac{1}{\left|S_{c}\right|} \sum_{(m, n) \in S_{c}} R_{(m, n), r e f},
\end{aligned}
$$

where, $R_{(m, n), r e f}, G_{(m, n), r e f}$, and $B_{(m, n), r e f}$ denote signal intensities of cells of the colour reference frame, and $\left|S_{c}\right|$ denotes the number of members of the set $S_{c}$. The complexity of caluclation for each refrence intensity is approximately $O\left(I^{(2)} \times j^{(2)}\right)$.

$\sigma_{R, r e f}^{c}, \sigma_{G, r e f}^{c}$, and $\sigma_{B, \text { ref }}^{c}$ are standard deviations for a colourc obtained from the colour reference frame. They are expressed as,

$$
\begin{aligned}
& \sigma_{R, r e f}^{c}=\sqrt{\frac{1}{\left|S_{c}\right|-1} \sum_{(m, n) \in S_{c}}\left(R_{r e f}^{c}-R_{(m, n), r e f}\right)^{2}}, \\
& \sigma_{G, r e f}^{c}=\sqrt{\frac{1}{\left|S_{c}\right|-1} \sum_{(m, n) \in S_{c}}\left(G_{r e f}^{c}-G_{(m, n), r e f}\right)^{2}}, \text { and } \\
& \sigma_{B, r e f}^{c}=\sqrt{\frac{1}{\left|S_{c}\right|-1} \sum_{(m, n) \in S_{c}}\left(B_{r e f}^{c}-B_{(m, n), r e f}\right)^{2}} .
\end{aligned}
$$

The complexity of caluclation for each standard deviation is approximately $O\left(\left(I^{(2)} \times j^{(2)}\right)^{2}\right)$.

\section{Performance Evaluation}

\subsection{EXPERIMENTAL CONDITIONS}

Assumptions of experiment is listed in Table 3. The communication distance denotes the distance between the LCD and the USB camera and is set at 150, 200, 250 and 300 [cm].

Table 3: Experimental Assumptions

\begin{tabular}{cc}
\hline \hline Environment & indoors, $830[\mathrm{Lux}]$ \\
\hline Communication distance & $150,200,250,300[\mathrm{~cm}]$ \\
\hline Resolution of the USB camera & $640 \times 480$ \\
\hline Frame rate of the LCD and the USB camera & $5.0[\mathrm{fps}]$ \\
\hline Number of trials & $1000[$ time $]$ \\
\hline \hline
\end{tabular}


The International Journal of Multimedia \& Its Applications (IJMA) Vol.9, No.2, April 2017

Table 4: Experimental Conditions

\begin{tabular}{|c|c|c|c|c|c|c|}
\hline & Ex1 & Ex2a & Ex2b & Ex3a & Ex3b & Ex3c \\
\hline $\begin{array}{c}\text { The number of } \\
\text { colours }\end{array}$ & $\begin{array}{c}8 \\
\text { (black, blue, } \\
\text { green, cyan, } \\
\text { red, magenta, } \\
\text { yellow, white) }\end{array}$ & \begin{tabular}{|c|}
4 \\
(black, \\
blue, red, \\
magenta)
\end{tabular} & $\begin{array}{c}2 \\
\text { (black, } \\
\text { red) }\end{array}$ & \begin{tabular}{|c|}
4 \\
(black, \\
blue, red, \\
magenta)
\end{tabular} & $\begin{array}{c}2 \\
\text { (black, } \\
\text { red) }\end{array}$ & $\begin{array}{c}8 \\
\text { (black, blue, } \\
\text { green, cyan, } \\
\text { red, magenta, } \\
\text { yellow, white) }\end{array}$ \\
\hline $\begin{array}{c}\text { The number of boxes } \\
\text { per frame }(M \times N)\end{array}$ & $\begin{array}{c}648 \\
(18 \times 36)\end{array}$ & $\begin{array}{c}648 \\
(18 \times 36) \\
\end{array}$ & $\begin{array}{c}648 \\
(18 \times 36) \\
\end{array}$ & $\begin{array}{c}968 \\
(22 \times 44) \\
\end{array}$ & $\begin{array}{c}1922 \\
(31 \times 62)\end{array}$ & $\begin{array}{c}1300 \\
(26 \times 50)\end{array}$ \\
\hline $\begin{array}{c}\text { The number of pixels } \\
\text { per box } \\
\text { (height } \times \text { width) }\end{array}$ & $\begin{array}{c}484 \\
(22 \times 22)\end{array}$ & $\begin{array}{c}484 \\
(22 \times 22)\end{array}$ & $\begin{array}{c}484 \\
(22 \times 22)\end{array}$ & $\begin{array}{c}324 \\
(18 \times 18)\end{array}$ & $\begin{array}{c}169 \\
(13 \times 13)\end{array}$ & $\begin{array}{c}256 \\
(13 \times 13)\end{array}$ \\
\hline $\begin{array}{l}\text { The number of } \\
\text { total pixels }\end{array}$ & 313,632 & 313,632 & 313,632 & 313,632 & 324,818 & 332,800 \\
\hline $\begin{array}{c}\text { FEC } \\
\text { (Code Rate) }\end{array}$ & $\begin{array}{l}\text { Uncoded } \\
(1.00)\end{array}$ & $\begin{array}{c}\text { Uncoded } \\
(1.00)\end{array}$ & $\begin{array}{c}\text { Uncoded } \\
(1.00)\end{array}$ & \begin{tabular}{|c|} 
Uncoded \\
$(1.00)$
\end{tabular} & $\begin{array}{c}\text { Uncoded } \\
(1.00)\end{array}$ & $\begin{array}{l}\text { Turbo coded } \\
(0.50)\end{array}$ \\
\hline $\begin{array}{c}\text { the number of } \\
\text { symbols per frame }\end{array}$ & 644 & 644 & 644 & 964 & 1918 & 1296 \\
\hline $\begin{array}{c}\text { the number of bits } \\
\text { per frame }\end{array}$ & 1932 & 1288 & 644 & 1928 & 1918 & 1942 \\
\hline
\end{tabular}

We define six experimental conditions namely Ex1, Ex2a, Ex2b, Ex3a, Ex3b, and Ex3c, as shown in Table 4. In order to maintain fairness of comparison, for all the six conditions, we use almost the same number of LCD pixels at the transmitter. The details of these conditions are listed below:

- By comparing the conditions Ex1, Ex2a and Ex2b, the transmission rate of Ex2a and Ex2b are smaller than that of Ex1. These three conditions have the same number of boxes and the size of the boxes is also the same. However, since, for Ex2a, only two colours are used for transmission, the achievable transmission rate is two-thirds of that of Ex1. Similarly, the rate of Ex $2 b$ is one-third of that of Ex1 because the condition Ex2b uses only one colour. Since the number of boxes in one frame is $M \times N-4$, the number of bits in one frame of Ex1, Ex2a, and Ex2b is 1932, 1228, and 644 [bits], respectively.

- By comparing the conditions Ex1, Ex3a, and Ex3b, they have almost the same transmission rate.

For Ex3a, although it uses only two colours, the number of boxes is $3 / 2$ times that of Ex 1 in order to achieve almost the same transmission rate. Similarly, the number of boxes of Ex $3 b$ is 3 times that of Ex1 because Ex $3 b$ uses only one colour.

- The condition Ex3c introduces Turbo codes. The specification of the turbo code used in this paper is shown in Table 5. Since the code rate of the Turbo code is $1 / 2$, we set the number of boxes of Ex3c is twice that of Ex1. By this setting, the transmission rate of Ex3c is almost the same as that of Ex1.

For easily understanding, examples of a displayed signal on the LCD for these experiment conditions are shown in Fig. 7. 


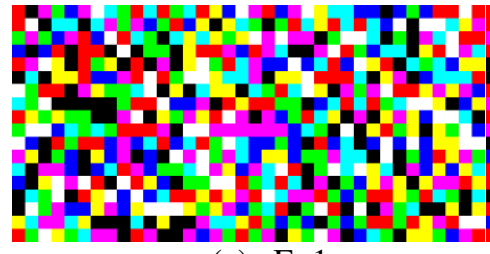

(a) Ex1

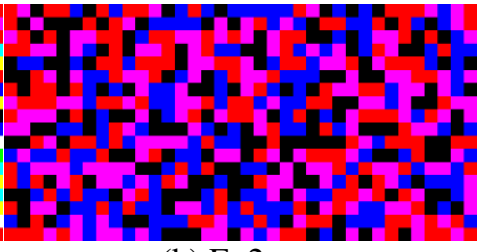

(b) Ex2a

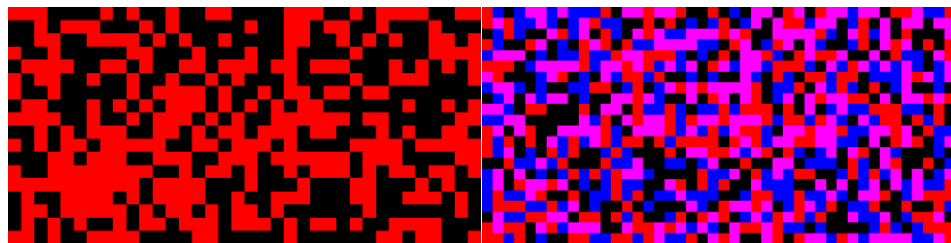

(c)Ex2(b)

(d) Ex3a

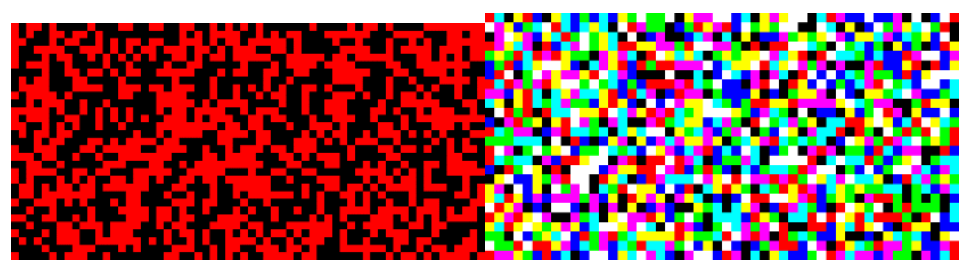

(e) Ex3b

(f) $\mathrm{Ex} 3 \mathrm{c}$

Figure 7: Examples of displayed images for each condition

Table 5: Parameters of the Turbo Code

\begin{tabular}{cc}
\hline \hline Constraint length & 3 \\
\hline Generator matrix & $(7,5)$ \\
\hline Coded rate & 0.50 \\
\hline Word length & Information:1522[bit] \\
& Coded word:3048[bit] \\
\hline Decoding iterations & $10[$ time] \\
\hline
\end{tabular}

\subsection{EXPERIMENTAL RESULTS}

The constructed prototype system is evaluated in terms of BER performance, FER performance, and normalized throughput performance. The throughput performance is normalized by the achievable maximum throughput of the condition Ex1 by error-free communication.

\subsubsection{Performance CoMparison BETWEen Ex2A, EX2B AND Ex1}

Figure 8 shows BER performance in terms of the communication distance. From the figure, we can find that BER performance degrades as increase of the communication distance. We can find that the conditions Ex1, Ex2a and Ex2b exhibit almost the same BER performance. FER performance of these conditionscan be also almost the same as shown in Fig. 9. From the result, we understand that three primary colours can be considered mutually orthogonal. Therefore, a system using the three colours can achieve better performance compared with a system using only one colour or two colours. The throughput performance in Fig. 10 clearly shows effectiveness of CSK when the communication distance is $200[\mathrm{~cm}]$ or less. 


\subsubsection{Performance CoMparison between Ex3A, Ex3B, Ex3C AND Ex1}

Figures 11 and 12 show BER performance and FER performance of the conditions Ex1, Ex3a, Ex3b and Ex3c. By comparing the performance between Ex1, Ex3a, and Ex3b, we can find that the condition Ex1 exhibits superior performance than the conditions Ex3a and Ex3b. As shown in Table 4, the conditions Ex3a and Ex3b uses only two or one colours. In order to achieve almost the same bit rate as the condition Ex1, the conditions Ex3a and Ex3b should increase the number of boxes by decreasing their box size. We infer that the decrease of the box size causes the performance degradation.

From Fig. 11, by the condition Ex3c, we note that error-free communication can be achieved when the communication distance is $200[\mathrm{~cm}]$ or less. Although the size of boxes of the condition Ex3c is less than that of Ex1, the performance of Ex3c is superior to that of Ex1 by the effect of error correction. By observing Fig. 13, the condition Ex3c achieves almost the maximum throughput when the communication distance is $250[\mathrm{~cm}]$ or less.

\subsubsection{PERFORMANCE COMPARISON BETWEen EX2A, EX2B, EX3A AND EX3B}

The number of colours of the conditions Ex2a and Ex3a is the same, i.e., two. From Figs. 8 and 11, BER performance of Ex2a is better than that of Ex3a because the size of cells of Ex2a is bigger than that of Ex3a. Therefore, from Figs. 10 and 13, although the number of cellsof Ex3a is larger than that of Ex2a, we can obtain better throughput performance by Ex2a in comparison with that of Ex3a. The same characteristics is also obtained by comparing Ex3b with Ex2b. From the performance comparison, high throughput performance can be obtained by enlarging the size of cells in the case of large communication distance.

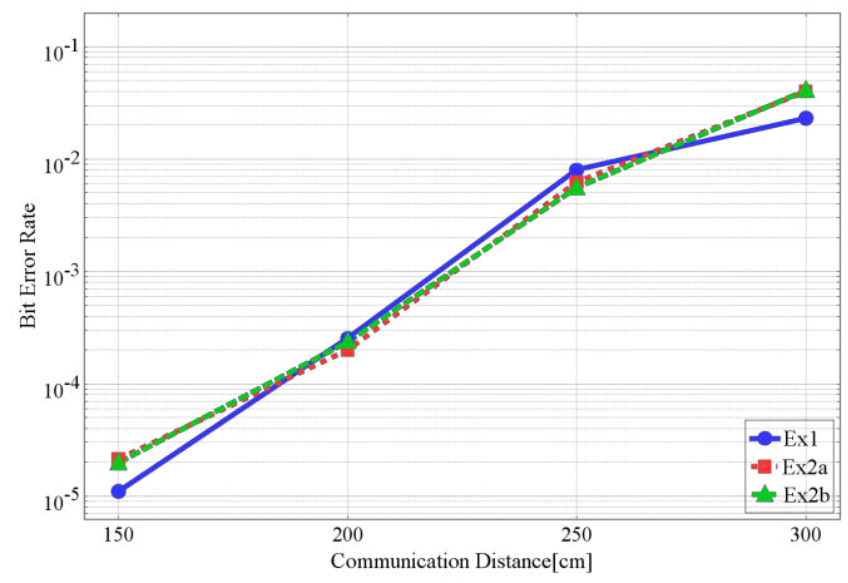

Figure 8: BER performance of Ex2a, Ex2b and Ex1 
The International Journal of Multimedia \& Its Applications (IJMA) Vol.9, No.2, April 2017

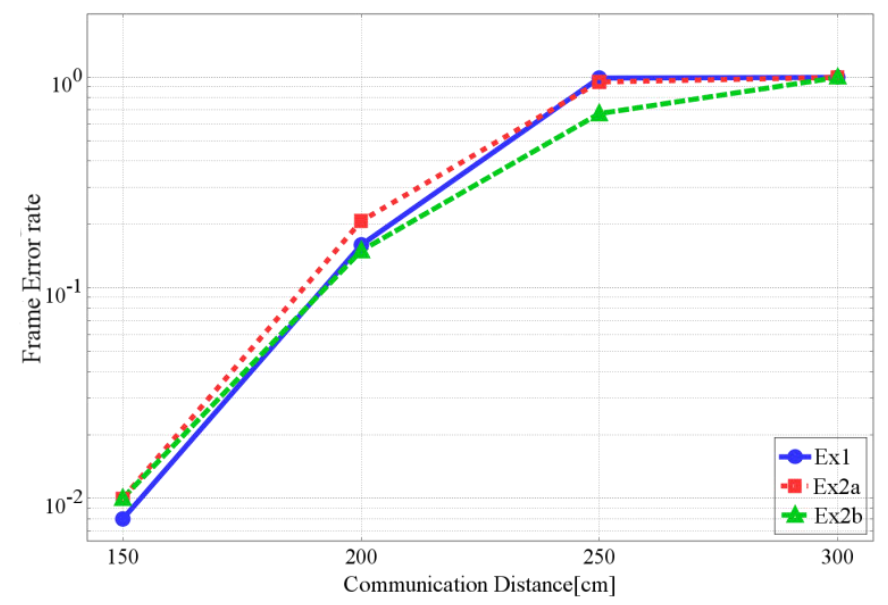

Figure 9: FER performance of Ex2a, Ex2b and Ex1

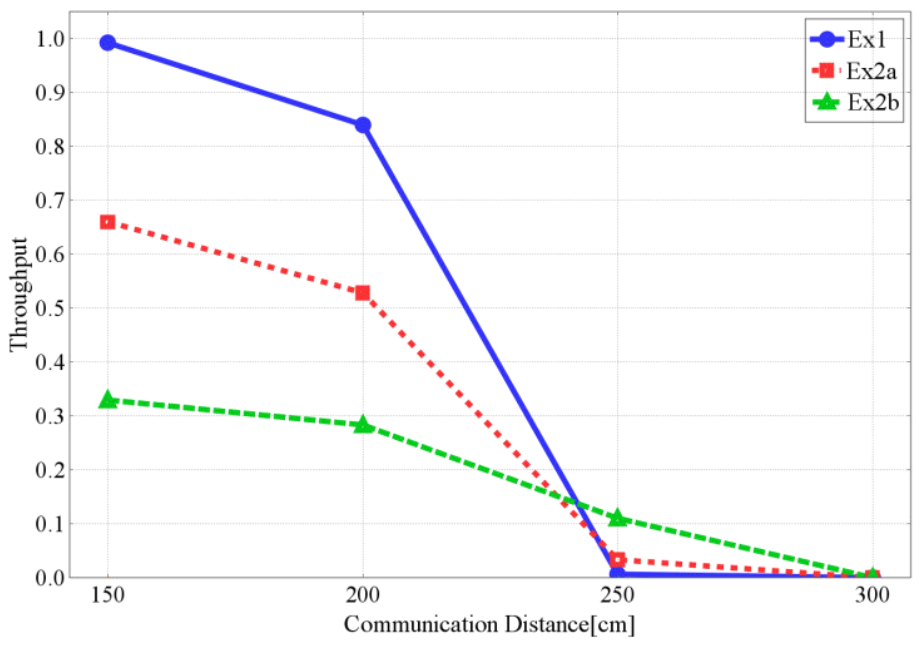

Figure 10: Normalized throughput performance of Ex2a, Ex2b and Ex1

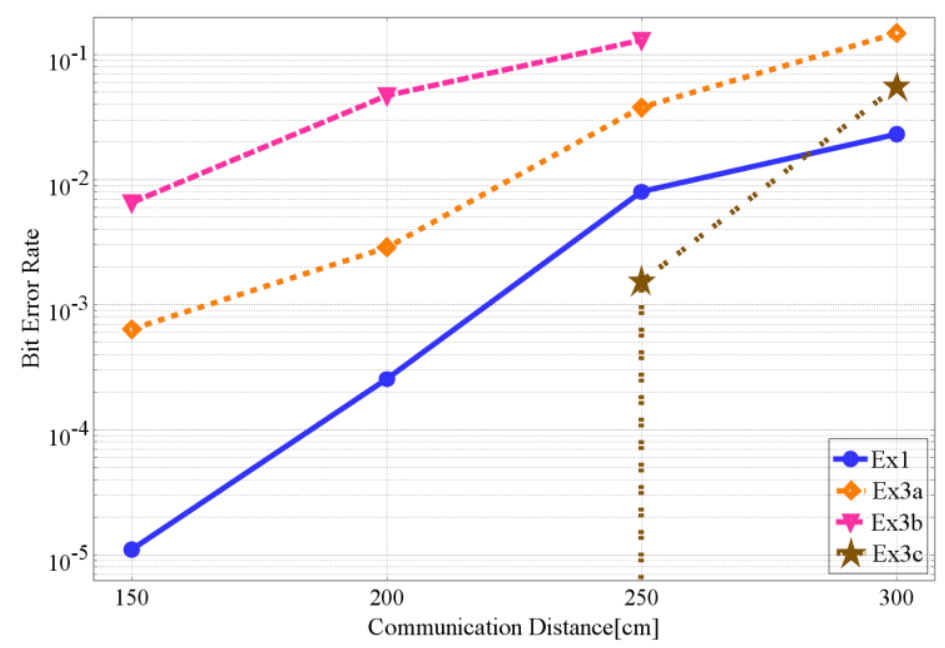

Figure 11: BER performance of Ex3a, Ex3b, Ex3c and Ex1 
The International Journal of Multimedia \& Its Applications (IJMA) Vol.9, No.2, April 2017

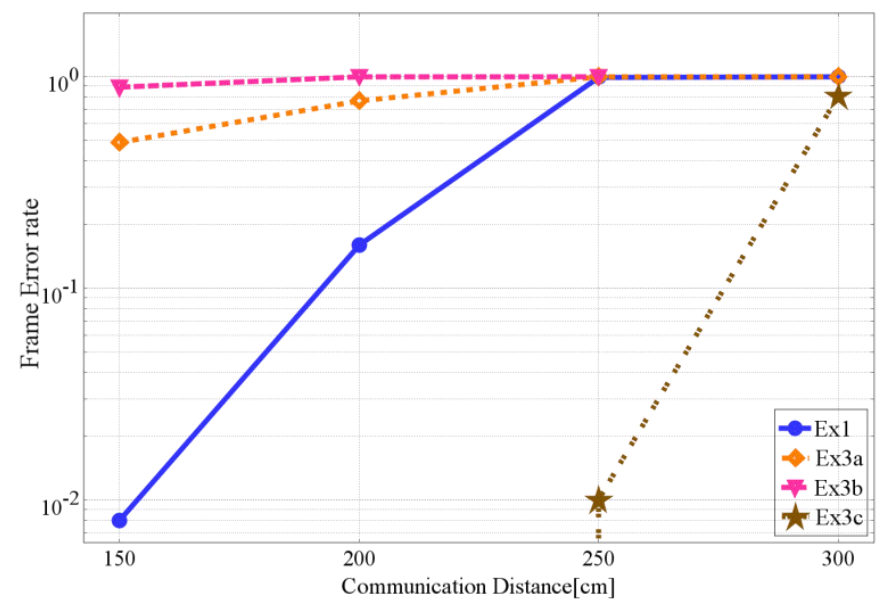

Figure 12: FER performance of Ex3a, Ex3b, Ex3c and Ex1

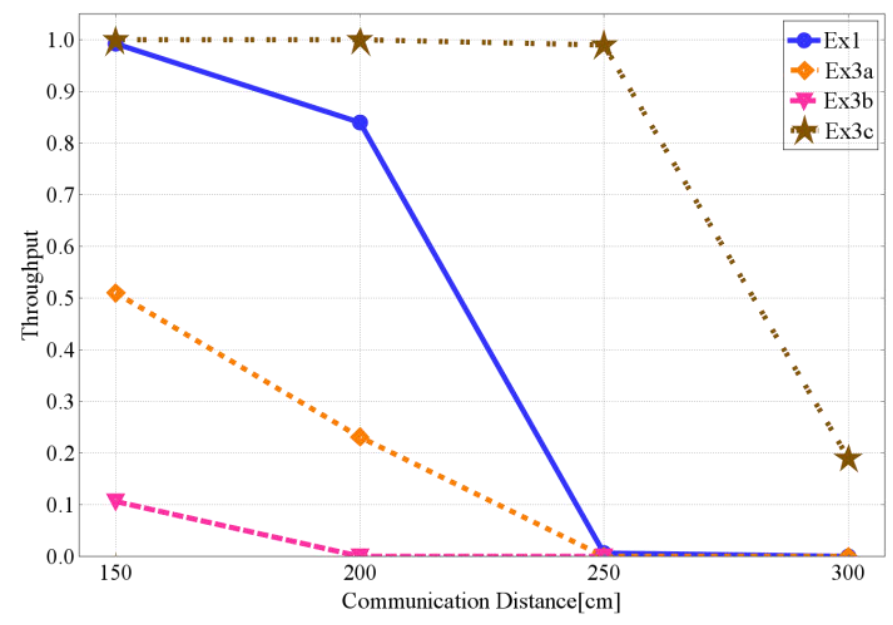

Figure 13: Normalized throughput performance of Ex3a, Ex3b, Ex3c and Ex1

\section{Conclusions}

In this paper, we have constructed a prototype of PT-VLC systems based on an LCD and an IS. We have proposed a new CSK algorithm and integrated it to the prototype. By the result of experiment, we have shown that the proposed algorithm achieves high throughput by using property of CSK. We have also utilized a Turbo code for error correction and shown effectiveness of it in the proposed VLC system. For a future work, we need to be study alternative schemes to reduce complexity of calculations for colour estimation and to increase the number of displayed colours to improve a transmission rate.

\section{REFERENCES}

[1] S. Haruyama, "Advances in visible light communication technologies," 2012 38th European Conference and Exhibition on Optical Communications, 2012

[2] H. Elgala, R. Mesleh, H. Haas, "Indoor Optical Wireless Communication: Potential and State-of-theArt," IEEE Communications Magazine, pp.56-62, 2011

[3] IEEE 802.15 Working Group for Wireless Specialty Networks (WSN), http://www.ieee802.org/15/

[4] A. M. Cailean, M. Dimian, "Impact of IEEE 802.15.7 Standard on Visible Light Communications Usage in Automotive Applications," IEEE Communications Magazine, pp.2-7, 2017 
The International Journal of Multimedia \& Its Applications (IJMA) Vol.9, No.2, April 2017

[5] Y. Pan, J. Wu, C. Long, "MLCS: Face-to-Face Mobile-LCD Communication System based on Visible Light Communication," Proc. IEEE Wireless and Communications and Networking Conference, pp. 3224-3229, 2014

[6] S. Hranilovic, F.R. Kschischang, "Short-Range Wireless Optical Communication using Pixelated Transmitters and Imaging Receivers," Proc. IEEE International Conference on Communications, pp.891-895, 2004

[7] T. Fujihashi, T. Koike-Akino, P. V. Orlik, T. Watanabe, "Experimental Throughput Analysis in Screen-Camera Visual MIMO Communications, Proc. 2016 IEEE Global Communications Conference, 2016

[8] J. Amano, T. Wada, and K. Mukumoto, "An Evaluation of a Parallel Transmission Visible Light Communication System employing an LDPC code,'Proc. International Symposium on Information Theory and its Applications(ISITA 2012), pp. 566-570, 2012.

[9] T. Wada, H. Furukawa, K. Mukumoto, "A study on parallel transmission wireless visible light communications using color shift keying," Proc. 2014 IEEE Asia Pacific Conference on Circuits and Systems, 2014

[10] C. E. Mejia, C. N. Georghiades, Y. H. Al-Badarneh, "Code Design in Visible Light Communications Using Color-Shift-Keying Constellations, 2016 IEEE Global Communications Conference, 2016

[11] N. Murata, Y. Kozawa, Y. Umeda, "Digital Color Shift Keying With Multicolor LED Array,” IEEE Photonics Journal, Vol 8, No 4, 2016

[12] P. M. Butala, H. Elgala, T. D. C. Little, "Performance of Color Shift Keying under Non-Linear System Model and Illumination Constraints," 2015 IEEE Globecom Workshops, 2015

[13] S. Igata, Y. Kozawa, Y. Umeda, H. Habuchi, "BER performance of digital color shift keying with target color control," 2016 10th International Conference on Signal Processing and Communication Systems, 2016

[14] Casio Picalico, http://picalico.casio.com/en/ (accessed April, 2017)

[15] A. Goldsmith, Wireless Communications, Cambridge University Press, 2005

\section{Authors}

Tadahiro WADA received the B.E. and M.E. and Ph.D. degrees in electronics and informationengineering from Nagoya University, Japan, in 1993, 1995, and 1998, respectively.Since 1998, he has been with the Faculty of Engineering, Shizuoka University, Hamamatsu, Japan, where he was firstly an assistant professor, and is currently an associate processor. From 2004 to 2005, he was a visiting researcher atthe University of Sydney, Australia.His current research interests are wireless optical communications,cooperative

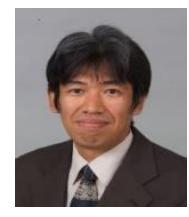
networks, error control techniques, and meteor burst communications.

Haruki FURUKAWA received the B.E. and M.E. degrees in electric and electronics engineering fromShizuoka University, in 2013 and 2015, respectively.His research interestswere wireless optical communications and error correction codes.

Kaiji Mukumoto received the Ph.D. degree in electrical engineering from Shizuoka University, Hamamatsu, Japan, in 1993. He is currently with the Division of Technical Service, Shizuoka Uni versity, as a technical pecialist. His current research interest includes communication theory, software radios, wireless sensor networks, and meteor burst communications.

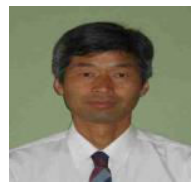

